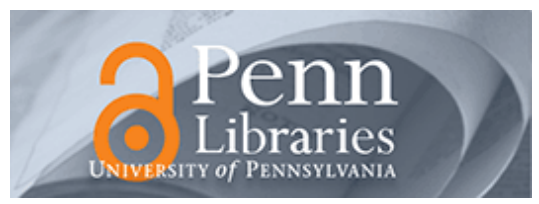

University of Pennsylvania ScholarlyCommons

$1-22-2009$

\title{
Strong Coupling of the Fe-Spin State and the As-As Hybridization in Iron-Pnictide Superconductors from First-Principle Calculations
}

Taner Yildirim

National Institute of Standards and Technology; University of Pennsylvania, taner@seas.upenn.edu

Follow this and additional works at: https://repository.upenn.edu/mse_papers

Part of the Materials Science and Engineering Commons

\section{Recommended Citation}

Yildirim, T. (2009). Strong Coupling of the Fe-Spin State and the As-As Hybridization in Iron-Pnictide Superconductors from First-Principle Calculations. Retrieved from https://repository.upenn.edu/ mse_papers/197

\section{Suggested Citation:}

Yildirim, T. (2009). "Strong Coupling of the Fe-Spin State and the As-As Hybridization in Iron-Pnictide Superconductors from First-Principle Calculations." Physical Review Letters. 102, 037003.

(C) 2009 The American Physical Society

http://dx.doi.org/10.1103/PhysRevLett.102.037003

This paper is posted at ScholarlyCommons. https://repository.upenn.edu/mse_papers/197

For more information, please contact repository@pobox.upenn.edu. 


\title{
Strong Coupling of the Fe-Spin State and the As-As Hybridization in Iron-Pnictide Superconductors from First-Principle Calculations
}

\author{
Abstract \\ From first-principles calculations, we unravel surprisingly strong interactions between arsenic ions in iron \\ pnictides, the strength of which is controlled by the Fe-spin state. Reducing the Fe-magnetic moment \\ weakens the Fe-As bonding, and in turn, increases As-As interactions, causing giant reduction in the $c$ \\ axis. For the $\mathrm{CaFe}_{2} \mathrm{As}_{2}$ system, this reduction is as large as $1.4 \AA$. Since the large $c$ reduction has been \\ recently observed only under high pressure, our results suggest that the iron magnetic moment should be \\ present in Fe-pnictides at all times at ambient pressure. Finally, the conventional electron-phonon \\ coupling in the collapsed phase of CaFe2As2 gives a maximum $T c$ of $0.6 \mathrm{~K}$ and cannot explain the $\sim 12 \mathrm{~K}$ \\ superconductivity observed in some experiments. Implications of these findings on the mechanism of \\ superconductivity in iron pnictides are discussed.

\section{Disciplines} \\ Engineering | Materials Science and Engineering

\section{Comments} \\ Suggested Citation: \\ Yildirim, T. (2009). "Strong Coupling of the Fe-Spin State and the As-As Hybridization in Iron-Pnictide \\ Superconductors from First-Principle Calculations." Physical Review Letters. 102, 037003. \\ (C) 2009 The American Physical Society \\ http://dx.doi.org/10.1103/PhysRevLett.102.037003
}




\title{
Strong Coupling of the Fe-Spin State and the As-As Hybridization in Iron-Pnictide Superconductors from First-Principle Calculations
}

\author{
T. Yildirim ${ }^{1,2, *}$ \\ ${ }^{1}$ NIST Center for Neutron Research, National Institute of Standards and Technology, Gaithersburg, Maryland 20899, USA \\ ${ }^{2}$ Department of Materials Science and Engineering, University of Pennsylvania, Philadelphia, Pennsylvania 19104, USA
}

(Received 4 August 2008; published 22 January 2009)

\begin{abstract}
From first-principles calculations, we unravel surprisingly strong interactions between arsenic ions in iron pnictides, the strength of which is controlled by the Fe-spin state. Reducing the Fe-magnetic moment weakens the Fe-As bonding, and in turn, increases As-As interactions, causing giant reduction in the $c$ axis. For the $\mathrm{CaFe}_{2} \mathrm{As}_{2}$ system, this reduction is as large as $1.4 \AA$. Since the large $c$ reduction has been recently observed only under high pressure, our results suggest that the iron magnetic moment should be present in Fe-pnictides at all times at ambient pressure. Finally, the conventional electron-phonon coupling in the collapsed phase of $\mathrm{CaFe}_{2} \mathrm{As}_{2}$ gives a maximum $T_{c}$ of $0.6 \mathrm{~K}$ and cannot explain the $\sim 12 \mathrm{~K}$ superconductivity observed in some experiments. Implications of these findings on the mechanism of superconductivity in iron pnictides are discussed.
\end{abstract}

DOI: 10.1103/PhysRevLett.102.037003

The recent discovery of superconductivity at $T_{c}$ 's up to $55 \mathrm{~K}$ in iron pnictide systems [1-5] has sparked enormous interest in this class of materials. So far, two types of materials have been discovered. The first one is the rareearth pnictide oxide layered systems, REOFeAs, which is denoted as "1111" [2-6]. The second class is the so-called " 122 " systems with the chemical formula $\mathrm{MFe}_{2} \mathrm{As}_{2}(M=$ $\mathrm{Ca}, \mathrm{Sr}$, etc.) [6-10]. The 122 systems are simpler in terms of their crystal structure due to absence of REO layers. The crystal structure of $\mathrm{CaFe}_{2} \mathrm{As}_{2}$ is shown in the inset to Fig. 1.

The pressure-induced superconductivity in these system is particularly interesting [11-13] because it provides a new avenue for investigation of the mechanism of the high- $T_{c}$ superconductivity. Very recently, a high pressure neutron scattering study has reported that the $\mathrm{CaFe}_{2} \mathrm{As}_{2}$ system undergoes a surprising transition to a "collapsedtetragonal phase" (cT-phase) under applied pressure in which the $c$ parameter is reduced from 11.7 to $10.6 \AA$ [1]. Motivated by this interesting report, in this Letter, we present a detailed first-principles study of Fe-pnictides with many surprising results.

We discovered that the optimized $c$-lattice parameter of $\mathrm{CaFe}_{2} \mathrm{As}_{2}$ varies by about $1.5 \AA$ depending on the magnetic configuration considered. Such a giant coupling of spinstate of an ion with its lattice is almost unheard of and deserves detailed investigation. Reducing Fe-moment by about $1 / 2$ gives lattice parameters that are quite close to high pressure data [1]. Hence, the collapsed-phase does not necessarily mean the total loss of Fe magnetism as suggested in Ref. [1] but could be due to large reduction of the Fe-moment. We explain this strange behavior by noting that in iron pnictide systems there are significant As-As hybridization (both intra and interplane arsenic ions) whose strength is controlled by the Fe-spin state. Reducing the Fe-spin state reduces the $\mathrm{Fe}$-As bonding,
PACS numbers: 74.25.Jb, 67.30.hj, 74.25.Kc, 75.30.Fv

which in turn, increases the As-As bonding and causes the observed huge reduction in the lattice parameters. This effect is maximized in the case of Ca122 system due to close proximity of two arsenic ions in adjacent Fe-planes (see Fig. 1). We argue that since there is no observation of large $c$ axis reduction during the normal to superconducting phase transition in iron pnictides, the iron magnetic moment should be present at all times at ambient pressure. Otherwise, one should have seen a large reduction in the $c$ axis as recently reported from high-

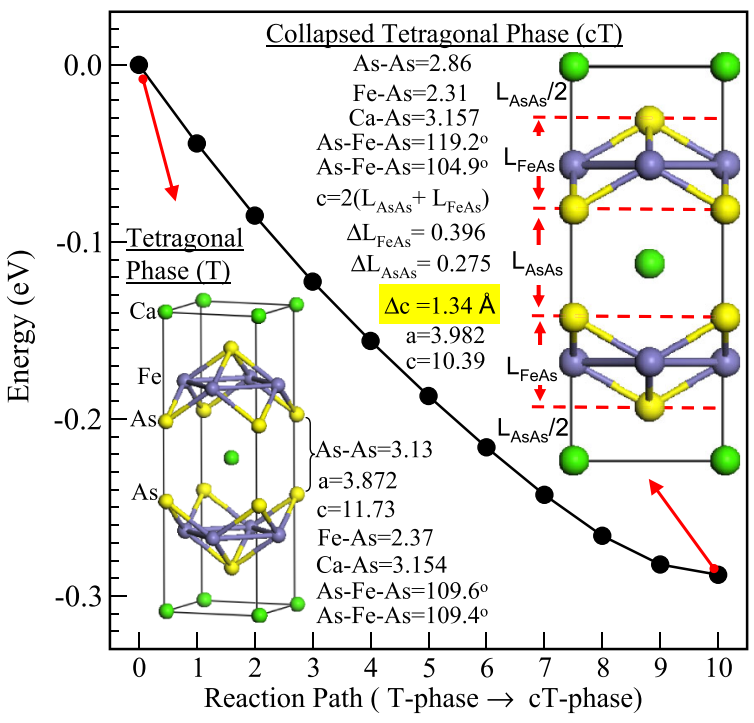

FIG. 1 (color online). Total energy as the Ca122 tetragonal phase goes to collapsed-tetragonal phase without any energy barrier during nonspin polarized structural optimization. The insets show the initial ( $T$-Phase) and final (cT-phase) Ca122 structures with relevant bond-distances (in $\AA$ ) and angles (in degrees). 
pressure neutron scattering experiments [1]. Hence, it seems that the Fe-magnetic moment in its paramagnetic state (i.e., no long-range order but nonzero Fe-site moment) is either required for the superconductivity or at least is not detrimental to the superconductivity.

The first-principles calculations were performed within the plane-wave implementation of the Perdew-BurkeErnzerhof (PBE) generalized gradient approximation (GGA) to density functional theory as implemented in the PWSCF package [14]. The details of the parameters used in the calculations can be found in Refs. $[15,16]$. In order to get a general understanding of the iron pnictides, in this study, we consider one example of each class of pnictides: namely $\mathrm{CaFe}_{2} \mathrm{As}_{2}$ for the 122 system with the smallest $\mathrm{Ca}$-ion available and the $\mathrm{BaFe}_{2} \mathrm{As}_{2}$ with the largest metal $\mathrm{Ba}$. For the 1111 system, we consider LaOFeAs. We also study a doped 122-system, i.e., $\mathrm{Na}_{0.5} \mathrm{Ca}_{0.5} \mathrm{Fe}_{2} \mathrm{As}_{2}$. Since in our $\sqrt{2} \times \sqrt{2}$-cell we have four chemical formulae, we consider a supercell where two $\mathrm{Na}$ and two $\mathrm{Ca}$ are ordered. For each given system, we have performed full structural optimization including the lattice parameters and the atomic positions. We consider our optimization is converged when the maximum force on each atom is less than $0.005 \mathrm{eV} / \AA$ and the pressure is less than $0.1 \mathrm{kbar}$. We have performed the full structural optimization for nonmagnetic (NM), i.e., "nonspin polarized," checkerboard antiferromagnetic (AF1) and stripe (AF2) spin configurations. Our results are summarized in Table I. As expected,

TABLE I. Various optimized structural parameters for NM, AF2, and AF1 spin configurations, respectively. The experimental data are taken from Refs. [8,17-19].

\begin{tabular}{|c|c|c|c|c|c|c|c|}
\hline & $\mathrm{a}$ & $\mathrm{b}$ & $\mathrm{c}$ & $\operatorname{As}(z)$ & $d_{\mathrm{FeAs}}$ & $M_{\mathrm{Fe}}$ & $E(\mathrm{meV})$ \\
\hline \multicolumn{8}{|c|}{$\mathrm{CaFe}_{2} \mathrm{As}_{2}$} \\
\hline NM & 5.63 & 5.63 & 10.39 & 0.36251 & 2.309 & 0 & 0.0 \\
\hline AF1 & 5.65 & 5.65 & 10.60 & 0.36440 & 2.338 & 1.3 & -16 \\
\hline AF2 & 5.61 & 5.48 & 11.61 & 0.36695 & 2.367 & 2.2 & -100 \\
\hline Exp. & 5.68 & 5.68 & 11.73 & 0.3665 & 2.370 & 1.0 & $\cdots$ \\
\hline \multicolumn{8}{|c|}{$\mathrm{Ca}_{0.5} \mathrm{Na}_{0.5} \mathrm{Fe}_{2} \mathrm{As}_{2}$} \\
\hline NM & 5.59 & 5.59 & 10.52 & 0.36284 & 2.31 & 0 & 0.0 \\
\hline $\mathrm{AF} 1^{\mathrm{a}}$ & 5.59 & 5.59 & 10.52 & 0.36284 & 2.31 & 0 & 0.0 \\
\hline AF2 & 5.43 & 5.53 & 12.05 & 0.36536 & 2.382 & 2.4 & -97 \\
\hline Exp. & 5.42 & 5.42 & 11.86 & $\cdots$ & $\cdots$ & 0.0 & $\cdots$ \\
\hline \multicolumn{8}{|c|}{$\mathrm{BaFe}_{2} \mathrm{As}_{2}$} \\
\hline NM & 5.58 & 5.58 & 12.45 & 0.3479 & 2.319 & 0 & 0.0 \\
\hline AF1 & 5.64 & 5.64 & 12.73 & 0.35231 & 2.382 & 2.1 & -80 \\
\hline AF2 & 5.70 & 5.59 & 12.83 & 0.3549 & 2.408 & 2.4 & -169 \\
\hline Exp. & 5.52 & 5.52 & 13.02 & 0.3545 & 2.397 & 1.0 & $\cdots$ \\
\hline \multicolumn{8}{|c|}{$\mathrm{LaOFeAs}$} \\
\hline NM & 5.64 & 5.64 & 8.59 & 0.35944 & 2.332 & 0 & 0.0 \\
\hline AF1 & 5.69 & 5.69 & 8.71 & 0.35128 & 2.393 & 2.1 & -86 \\
\hline AF2 & 5.67 & 5.73 & 8.72 & 0.34860 & 2.409 & 2.4 & -190 \\
\hline Exp. & 5.70 & 5.70 & 8.737 & 0.3479 & 2.407 & 0.35 & $\cdots$ \\
\hline
\end{tabular}

${ }^{\mathrm{a}}$ The AF1 configuration goes to $\mathrm{NM}$ for $\mathrm{Ca}_{0.5} \mathrm{Na}_{0.5} \mathrm{Fe}_{2} \mathrm{As}_{2}$. the ground state for all four systems is the stripe AF2 phase and the optimized parameters are in good agreement with the experimental data at ambient conditions.

The most striking and surprising finding listed in Table I is the giant dependence of the optimized $c$-lattice parameter on the spin configuration considered. For the case of $\mathrm{CaFe}_{2} \mathrm{As}_{2}$, we note that $\mathrm{AF} 1$ configuration is the next stable state (after the AF2), but the $c$-value is significantly reduced: $11.63 \AA$ versus $10.60 \AA$ for $\mathrm{AF} 2$ and $\mathrm{AF} 1$ spin configurations, respectively. The difference is even larger, when the Fe-magnetism is ignored (i.e., nonspin polarized calculations). The optimized $c$-value for NM-state is $10.39 \AA$, which is $1.34 \AA$ shorter than the experimental value at ambient pressure. We note that the optimized lattice parameters, $a=5.65 \AA$ and $c=10.6 \AA$ for the AF1 phase, are in reasonable agreement with the neutron data $(a=5.8 \AA$ and $c=10.6 \AA)$ [1]. Hence, based on this result, it is premature to conclude that the observed experimental cT-phase is due to the complete disappearance of the Fe-magnetism but as we shall see below, it is closely related to the magnitude of the $\mathrm{Fe}$-spin.

In Fig. 1, we show that the $T$-phase directly collapses into the cT-phase without any energy barrier if the Fe-magnetic moment is ignored. During the $c$ axis collapse, there is significant and comparable decrease in the height of the Fe-As and As-Ca-As planes, indicating that the whole lattice almost uniformly shrinks. Since there is no energy barrier between the $T$-phase and the cT-phase, as soon as we lose the Fe-magnetic moment for some reason, we should see the expected $c$-lattice reduction. Since this does not seem to happen in the superconducting samples, it is tempting to conclude that we have the Fe-moment present in the superconducting phase. This is a quite important result by itself to understand the mechanism of the high $T_{c}$ superconductivity observed in iron pnictides.

In order to get a better understanding of the cT-phase, we have performed electronic band structure calculations and studied the projected atomic density of states. Our results for the $T$-phase agrees well with previous calculations [2023]. Figure 2 shows that despite to the $1.4 \AA c$ reduction, the band structure and the PDOS of both phases are surprisingly similar. The main difference between the two phases is that most of the bands are shifted in lower energy in the cT-phase which is expected since the cT-phase has the lower energy. This downward shift of the bands is most obvious in the band structure plot along the $\Gamma-M$ direction as shown in Fig. 2. In the $T$-phase, there were several states above the Fermi level which crosses the $E_{F}$ along the $M-\Gamma$ direction. This results in a larger density of states at the Fermi level. However, for the case of cT-phase, we note that most of the bands just above $E_{F}$ in the $T$-phase are now just below the $E_{F}$ in the $c T$-phase, and there is only one band which crosses the $E_{F}$ along the $\Gamma-M$ direction. This explains why $N\left(E_{F}\right)$ is reduced in the cT-phase. The other and probably the most important difference is the change in 


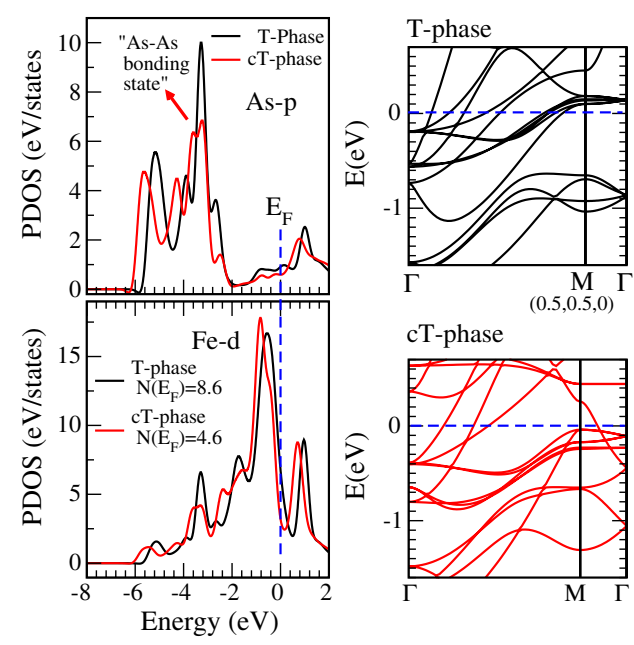

FIG. 2 (color online). The projected electronic density of states (PDOS) for As $p$-orbital (top-left) and Fe-d orbital (bottom left) for $T$ - and cT-phases. The right two panels show the band structure along $\Gamma-M-\Gamma$. The As-As $p_{z}$-bonding orbital is indicated by the arrow on the top left panel, and it is shown in Fig. 3.

the peak shape of the states near $3 \mathrm{eV}$ below $E_{F}$. Visual inspection of these orbitals indicate that there is significant As-As hybridization in this system. The As-As hybridization becomes more significant in the cT-phase. This observation is quite unexpected, and as we shall see below, it actually explains the mystery why the Fe-spin state has a huge effect on the As-z coordinates as first noted in [24].

In order to demonstrate that there is large hybridization between As ions in the Ca122 system, we show the contour plots of the relevant orbitals in Fig. 3. It is clear that the As ion below the top Fe-plane makes a bond (or hybridizes) with the arsenic ion which is above the lower Fe-plane. Hence, this overlap of the As-As along the
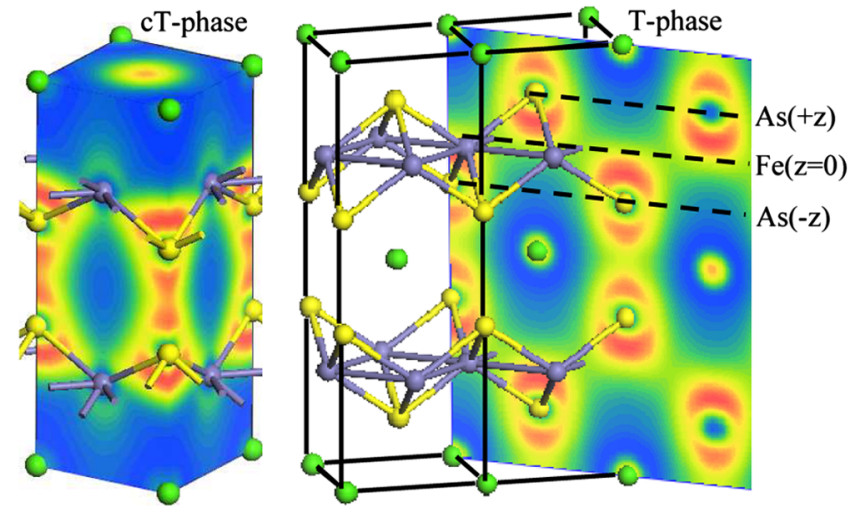

FIG. 3 (color online). Contour plot of the As-As $p_{z}$-hybridization orbital for cT-phase (left) and $T$-phase (right), respectively. Note that the As-As hybridization present in both phases is much more significant in the cT-phase. There is also clear hybridization between intra-As atoms below and above the Fe-plane in the $T$-phase (right). $c$ axis makes this system quite isotropic and far from being layered system. According to bond-population analysis, the As-As bond strength increased almost twice in the cT-phase. Because of close proximity of the As ions in adjacent Fe-layers, the observation of the As-As interaction is probably not that surprising. What is surprising is to see that there is almost the same type of hybridization between two arsenic ions on the same Fe-plane as shown in the right panel of Fig. 3.

Since we have shown that the As ion above the Fe-plane has a strong overlap with the As ion below the same iron plane, their interaction is automatically increased as the $\mathrm{Fe}$-As interaction decreases due to decrease in the Fe-moment which changes the chemistry of the Fe ion. Therefore, we have now a mechanism which explains why the As ion $z$ values get shorter with the decreasing Fe-moment. Our mechanism also explains why we see a smaller reduction in the $c$ axis for the LaOFeAs than the 122 system as listed in Table I. The reduction in the $c$ axis in the LaOFeAs system is due to the intraplane As-As interaction only since there are no two adjacent FeAs planes to interact to with each other as in the case of Ca122. Our theory also predicts that for larger ions like $\mathrm{Ba}$, we should see less $c$ reduction because the As-As distance between two adjacent planes is now larger due to larger ionic radius of $\mathrm{Ba}$. In Table $\mathrm{I}$, we also show that similar $c$ reduction with Fe-spin occurs in the doped $\mathrm{Na}_{0.5} \mathrm{Ca}_{0.5} \mathrm{Fe}_{2} \mathrm{As}_{2}$ system as well.

In order to convince the reader further that the spin-state of the iron is the key parameter that controls the As-As bonding in these systems, we have performed fixed-spin calculations for $\mathrm{CaFe}_{2} \mathrm{As}_{2}$, and the results are shown in Fig. 4. We consider ferro spin configuration to show that the spin-structure is a secondary effect, and the main effect is the on-site Fe-spin state. As we see from Fig. 4, when the Fe-moment is zero, the $c$ axis is the smallest consistent with the strongest As-As interaction (because As-Fe bonding is weak for nonmagnetic $\mathrm{Fe}$ ). As the $\mathrm{Fe}$ moment becomes significant, the Fe-As interaction gets stronger, and, therefore, the As-As distance starts to increase to optimize the $\mathrm{Fe}$-As bonding. Ironically, at the Fe-moment of $\mu_{B}=2.2$, we have the ideal tetragonal arrangement of the four As ion around each iron during which the two As-Fe-As angles are equal. At this point, the low- to high-Fe spin transition is obvious during which the a-axis is reduced while $c$ axis is increased significantly.

Since our results suggest that Fe-magnetism is either totally lost or reduced by half in the cT-phase, one wonders if the observed $\sim 12 \mathrm{~K}$ superconductivity in the vicinity of the collapse cT-phase of $\mathrm{CaFe}_{2} \mathrm{As}_{2}$ [13] can be explained by conventional electron-phonon (e-ph) coupling? In order to address this question, we have calculated phonon spectrum and Eliashberg function from linear response theory [14]. We used basically the same method and the equivalent parameters that are used in Ref. [25] for LaOFeAs. Our 


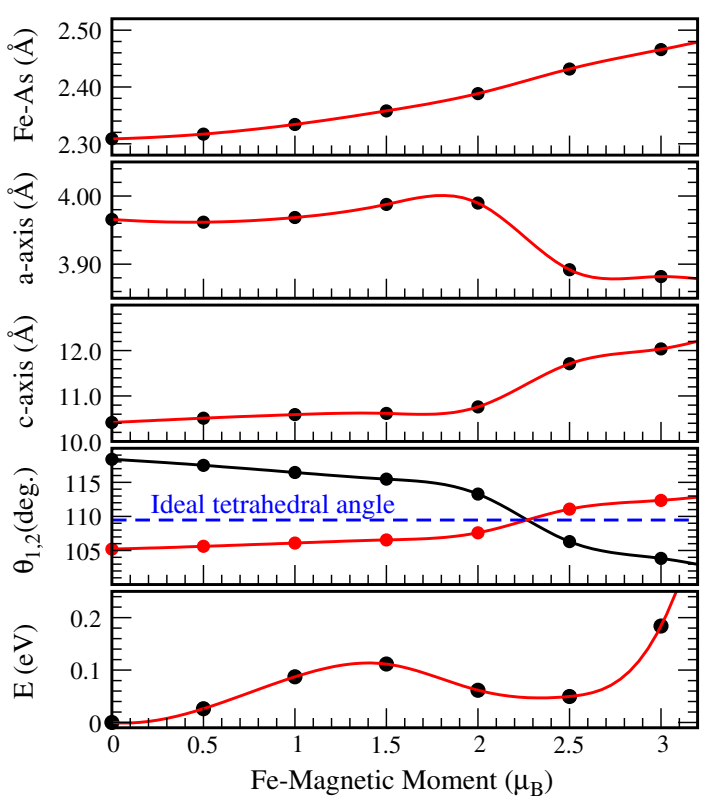

FIG. 4 (color online). Various structural parameters as a function of Fe-moment from fixed-spin calculations with ferromagnetic iron spin configuration.

results are summarized in Fig. 5 and very similar to those for LaOFeAs. We obtained a value of electron-phonon coupling $\lambda=0.23$ and the logarithmically average frequency $\omega_{\log }=218 \mathrm{~K}$, which gives $T_{c}=0.6 \mathrm{~K}$ using the Allen-Dynes formula with $\mu^{*}=0$ (i.e., an upper bound for $T_{c}$ ). Hence, the mechanism of superconductivity in the cT-phase of $\mathrm{CaFe}_{2} \mathrm{As}_{2}$ is likely unconventional, and probably, it is the same as in the other iron pnictides. This finding together with our results summarized in Table I suggests that in the cT-phase, the Fe-magnetism is not totally lost with pressure, but it is partially reduced. Hence, it seems that there is an optimum strength of Fe-spin state that is required for high- $T_{c}$ superconductivity.

In conclusion, we have revealed surprisingly strong As-As interactions in iron pnictides. The strength of this interaction is controlled by the Fe-As chemical bonding.

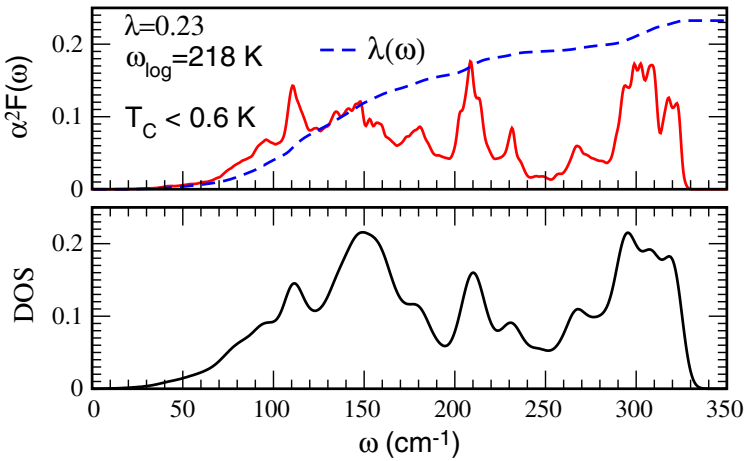

FIG. 5 (color online). Phonon density of states (DOS), Eliashberg function $\left[\alpha^{2} F(\omega)\right]$ and the frequency-dependent e-ph coupling $\lambda(\omega)$ (dashed line) for $\mathrm{CaFe}_{2} \mathrm{As}_{2}$ in the cT-phase.
Reducing the Fe-moment reduces the Fe-As bonding, which in turn increases the As-As interaction along the $z$-axis, causing arsenic atoms on opposite sides of Fe-square lattice to move towards each other. This explains the high sensitivity of the $z$-atom positions and the large reduction of the $c$ axis with the loss of Fe-magnetic moment. We showed that under external pressure, the high Fe-spin AF2 structure (i.e., stripe phase) should transform to a new structure with low $\mathrm{Fe}$-spin state and significantly reduced $c$ axis. We think that this could be the phase recently observed by high-pressure neutron scattering [1]. This is also consistent with our finding that the pressured induced $\sim 12 \mathrm{~K}$ superconductivity cannot be explained by the conventional e-ph coupling. The Fe-magnetism is still needed for an unconventional mechanism. Since at ambient pressure, we do not observe large $c$ axis drops in the superconducting samples, we conclude that the Fe-magnetic moment should be present at all times in these systems, at least in 122 materials such as $\mathrm{CaFe}_{2} \mathrm{As}_{2}$. The giant coupling of the on-site $\mathrm{Fe}$-magnetic moment with the As-As bonding that we have discovered here may provide a mechanism for the superconductivity.

The author acknowledges useful discussions with M. A. Green.

*taner@nist.gov

[1] A. Kreyssig et al., Phys. Rev. B 78, 184517 (2008).

[2] Y. Kamihara, T. Watanabe, M. Hirano, and H. Hosono, J. Am. Chem. Soc. 130, 3296 (2008).

[3] X. H. Chen et al., Nature (London) 453, 761 (2008).

[4] G. F. Chen et al., Phys. Rev. Lett. 100, 247002 (2008).

[5] Z. A. Ren, Mater. Res. Innovations 12, 105 (2008).

[6] C. Krellner et al., Phys. Rev. B 78, 100504(R) (2008).

[7] A. Jesche et al., Phys. Rev. B 78, 180504(R) (2008).

[8] A. I. Goldman et al., Phys. Rev. B 78, 100506(R) (2008).

[9] J. Zhao et al., Phys. Rev. B 78, 140504(R) (2008).

[10] Q. Huang et al., Phys. Rev. Lett. 101, 257003 (2008).

[11] T. Park et al., J. Phys. Condens. Matter 20, 322204 (2008).

[12] P. L. Alireza et al., J. Phys. Condens. Matter 21, 012208 (2009).

[13] M. S. Torikachvili, Phys. Rev. Lett. 101, 057006 (2008).

[14] http://www.pwscf.org.

[15] T. Yildirim, Phys. Rev. Lett. 101, 057010 (2008).

[16] T. Yildirim, arXiv:0807.3936.

[17] C. de la Cruz, Q. Huang, J. W. Lynn, J. Li, W. Ratcliff, II, H. A. Mook, G.F. Chen, J. L. Luo, N. L. Wang, and Pengcheng Dai, Nature (London) 453, 899 (2008).

[18] G. Wu et al., J. Phys. Condens. Matter 20, 422201 (2008).

[19] Q. Huang et al., Phys. Rev. Lett. 101, 257003 (2008).

[20] I. I. Mazin et al., arXiv:0806.1869v2.

[21] V. Vildosola, Phys. Rev. B 78, 064518 (2008).

[22] D. J. Singh and M.-H. Du, Phys. Rev. Lett. 100, 237003 (2008).

[23] D. J. Singh, Phys. Rev. B 78, 094511 (2008).

[24] Z. P. Yin et al., Phys. Rev. Lett. 101, 047001 (2008).

[25] L. Boeri et al., Phys. Rev. Lett. 101, 026403 (2008). 